\title{
Melanocortin 4 receptor stimulation prevents antidepressant-associated weight gain in mice caused by long-term fluoxetine exposure
}

\author{
María José Ortuño, ${ }^{1}$ Marc Schneeberger, ${ }^{2}$ Anoj Ilanges, ${ }^{2}$ François Marchildon, ${ }^{3}$ Kyle Pellegrino, ${ }^{2}$ Jeffrey M. Friedman, ${ }^{2}$ \\ and Patricia Ducy ${ }^{4}$
}

'Department of Cenetics and Development, College of Physicians and Surgeons, Columbia University, New York, New York, USA. ²aboratory of Molecular Cenetics, Howard Hughes Medical Institute and ${ }^{3}$ Laboratory of Molecular Metabolism, The Rockefeller University, New York, New York, USA. ${ }^{4}$ Department of Pathology and Cell Biology, College of Physicians and Surgeons, Columbia University, New York, New York, USA

\begin{abstract}
Contrasting with the predicted anorexigenic effect of increasing brain serotonin signaling, long-term use of selective serotonin reuptake inhibitor (SSRI) antidepressants correlates with body weight (BW) gain. This adverse outcome increases the risk of transitioning to obesity and interferes with treatment compliance. Here, we show that orally administered fluoxetine (Flx), a widely prescribed SSRI, increased BW by enhancing food intake in healthy mice at 2 different time points and through 2 distinct mechanisms. Within hours, Flx decreased the activity of a subset of brainstem serotonergic neurons by triggering autoinhibitory signaling through 5-hydroxytryptamine receptor 1a (Htr1a). Following a longer treatment period, Flx blunted 5-hydroxytryptamine receptor $2 \mathrm{c}(\mathrm{Htr} 2 \mathrm{c}$ ) expression and signaling, decreased the phosphorylation of cAMP response element-binding protein (CREB) and STAT3, and dampened the production of pro-opiomelanocortin (POMC, the precursor of $\alpha$-melanocyte stimulating hormone [ $\alpha-\mathrm{MSH}])$ in hypothalamic neurons, thereby increasing food intake. Accordingly, exogenous stimulation of the melanocortin 4 receptor (Mc4r) by cotreating mice with Flx and lipocalin 2, an anorexigenic hormone signaling through this receptor, normalized feeding and BW. FIx and other SSRIs also inhibited CREB and STAT3 phosphorylation in a human neuronal cell line, suggesting that these noncanonical effects could also occur in individuals treated long term with SSRIs. By defining the molecular basis of long-term SSRI-associated weight gain, we propose a therapeutic strategy to counter this effect.
\end{abstract}

\section{Introduction}

Selective serotonin reuptake inhibitors (SSRIs) are the most widely prescribed antidepressants in Western countries. Chief among them is fluoxetine (Flx), the active compound in Prozac, whose number of prescriptions in the United States reached over 25 millions in 2018 (1). SSRIs increase serotonin in the synaptic cleft by preventing reuptake by the serotonin transporter (SERT) in presynaptic neurons, leading to enhanced signaling in postsynaptic neurons $(2,3)$. Given the well-described anorectic effect of brain serotonin signaling $(4,5)$, SSRIs would be predicted to decrease food intake. Indeed, early studies have shown that rats injected with Flx lose weight $(6,7)$. Moreover, in the first months of treatment, some individuals on SSRI treatment lose weight, a manifestation consistent with the predicted tempering effect of enhanced central serotonin signaling on the regulation of feeding $(8,9)$. However, a growing number of clinical studies are reporting that long-term use ( $\geq 1$ year) of SSRIs is instead associated with weight gain (10-15). This paradoxical

Conflict of interest: The authors have declared that no conflict of interest exists. Copyright: @ 2021, American Society for Clinical Investigation.

Submitted: June 2, 2021; Accepted: October 19, 2021; Published: December 15, 2021 Reference information: / Clin Invest. 2021;131(24):e151976.

https://doi.org/10.1172/JCl151976. weight gain, which ranks as the third most common side effect of these drugs by those who use them (16), has become a major concern for clinicians, since it not only increases the long-term risk of metabolic syndrome but is also one of the most common reasons for premature discontinuation of the treatment $(12,13$, 15). Given that SSRIs are increasingly prescribed and that more than one-third of patients use them for longer than 2 years, this concern continues to mount $(16,17)$ (https://www.cdc.gov/nchs/ products/databriefs/db283.htm). Hence, there is a strong interest in understanding the biological basis of this paradoxical side effect to define therapeutic strategies to counter it.

\section{Results}

Both short- and a long-term treatments with oral Flx increase food intake. To investigate the mechanism that causes weight gain following long-term treatment with SSRIs, we analyzed the effect of Flx, one of the most prescribed SSRIs worldwide. We mimicked SSRI administration in humans by providing Flx orally to WT healthy mice at a dose that resulted in plasma concentrations comparable to the therapeutic levels achieved in humans (ref. 18 and Supplemental Figure 1A; supplemental material available online with this article; https://doi.org/10.1172/JCI151976DS1). Under these conditions, mice gained a significant amount of weight and adiposity compared with vehicle-treated controls following 
A. Vehicle
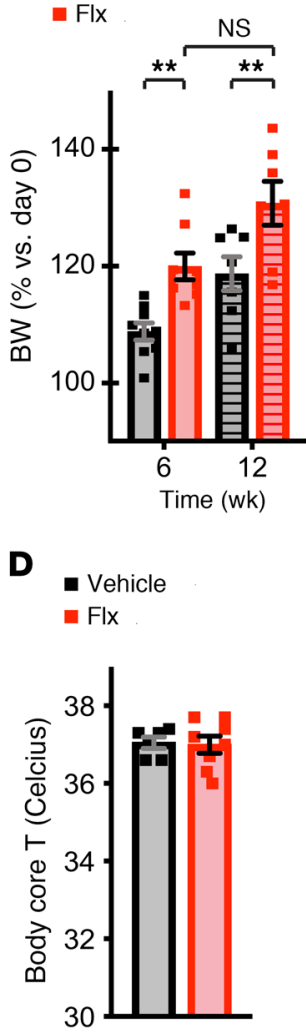

B

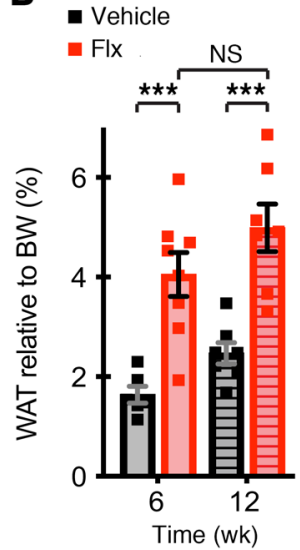

E
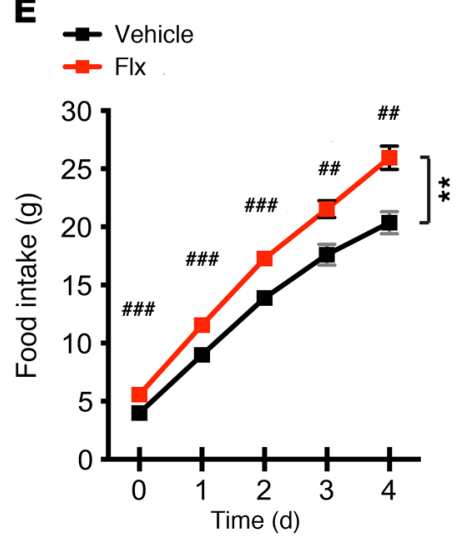

C - Vehicle

FIx
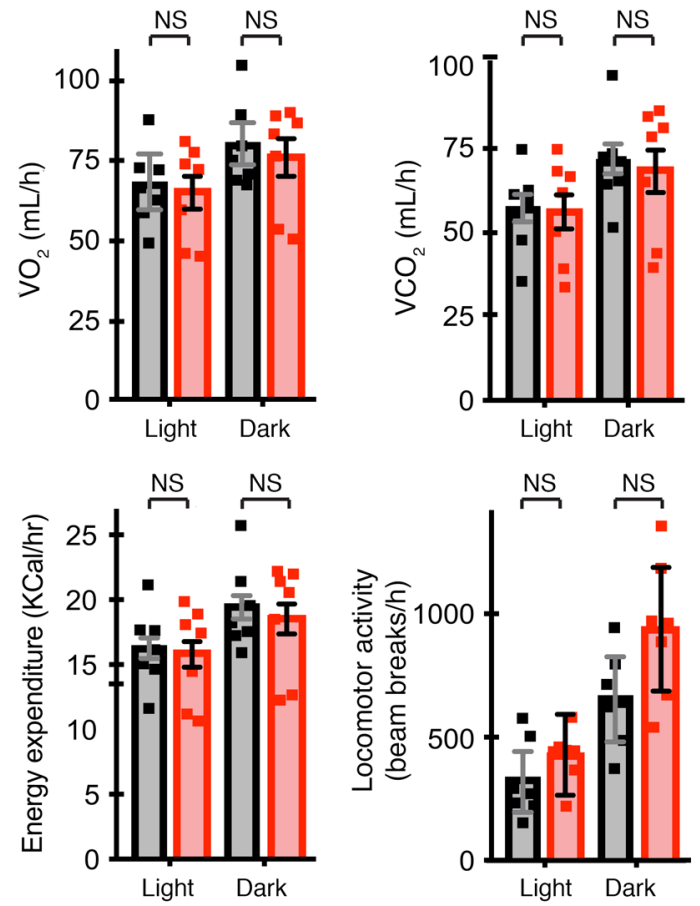

Figure 1. Long-term oral treatment with Flx increases BW and adiposity by specifically enhancing food intake. (A) Percentage of BW relative to day 0 in WT female mice treated with vehicle or Flx for 6 weeks (plain gray bars, $n=8-9$ mice/group, representative of 6 independent experiments) or 12 weeks (hatched bars, $n=7$ mice/group). (B) Percentage of WAT content relative to BW at the end of the 6-week ( $n=6-8$ mice/group) and 12-week ( $n=7$ mice/ group) treatment periods. (C and E) WT females treated with vehicle or Flx for 6 weeks. (C) Energy expenditure measured by V $\mathrm{O}_{2}, \mathrm{VCO}_{2}$, heat production, and locomotor activity recorded over the last 3 days of treatment ( $n=7$ mice/group). (D) Core body temperature (T) at the end of the treatment period ( $n=6-8$ mice/group). (E) Cumulative food intake measured over the last 5 days of treatment ( $n=6-7$ mice/group; data are representative of 2 independent experiments). Values represent the mean \pm SEM. ${ }^{* *} P \leq 0.01$ and ${ }^{* * *} P \leq 0.001$, by 2-way ANOVA followed by Šidák's test (A and B, locomotion activity


versus vehicle, analyzed for each time point.

a 6-week-long treatment (Figure 1, A and B, and Supplemental Figure 1, B-D and F). Lengthening the treatment to 12 weeks in another group of mice did not modify these parameters (Figure 1, A and B, and Supplemental Figure 1, E and F). To define which variables in the energy balance equation were responsible for this weight gain, we assessed the influxes (feeding) and outfluxes (energy expenditure) of energy in mice treated with Flx for 6 weeks. We found that none of the parameters associated with energy expenditure, i.e., basal metabolic rate, thermogenesis, or physical activity, was changed by the Flx treatment (Figure 1, C and D). In contrast, daily averaged and cumulative food intake over the previous 5 days was significantly higher in Flx-treated mice compared with vehicle-treated mice (Figure 1E and Supplemental Figure $1 G)$. When adjusted to body weight (BW), Flx treatment also resulted in a significant increase in cumulative food intake, whereas daily food intake trended upward but did not reach significance (Supplemental Figure 1, H and I). These results support the notion that, in mice, the increased BW caused by long-term oral treatment with Flx was maintained by increased food intake.
In the course of our analysis, we noticed that BW, fat mass, and cumulative food intake were already increased after 5 days of treatment with oral Flx in mice of both sexes (Figure 2, A-C, and Supplemental Figure 2, A-G). A day-by-day analysis revealed that treatment with Flx increased feeding daily and as early as within the first 24 hours (Figure 2D and Supplemental Figure $2 \mathrm{H}$ ). More specifically, a 14-hour treatment during the active (dark) phase of the day was sufficient to trigger a significant increase in food intake compared with that observed in vehicle-treated control mice (Figure 2E and Supplemental Figure 2I). As with the long-term treatment, averaged metabolism, thermogenesis, and physical activity were not changed by this acute Flx treatment in male and female animals (Figure 2, F and G, and Supplemental Figure $2 \mathrm{~J}-\mathrm{M})$. A day-by-day analysis of female mice also revealed no difference prior to BW/adiposity divergence between the vehicle- and Flx-treated groups (Supplemental Figure 2, $\mathrm{N}$ and $\mathrm{O}$ ). Thus, when given orally to mice, Flx caused weight gain by increasing food intake in an acute manner as well as in a long-term mode. 
A

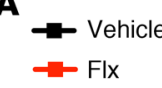

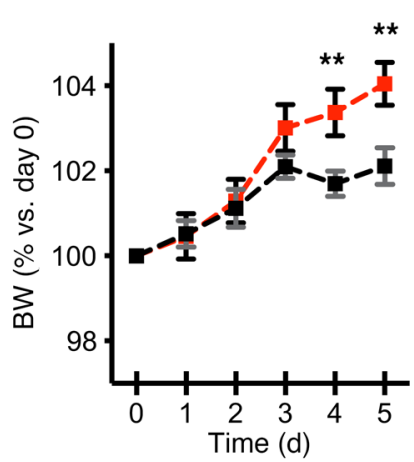

$\mathbf{E}$



B
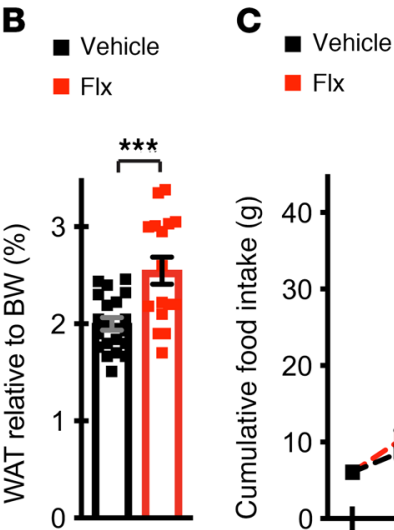

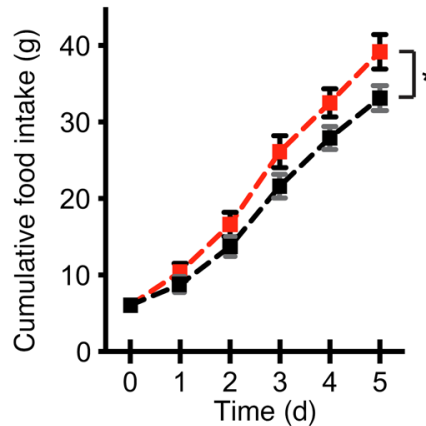

D

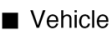

FIX

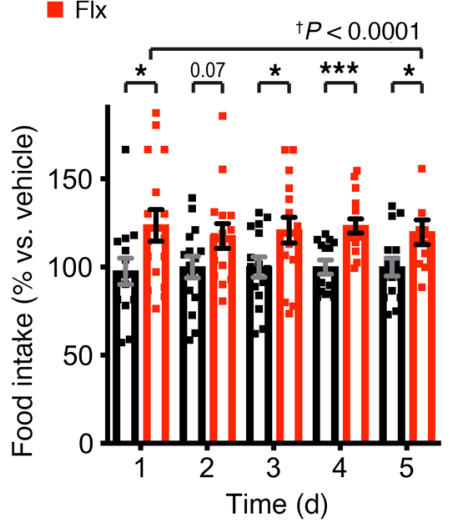

G - Vehicle alx
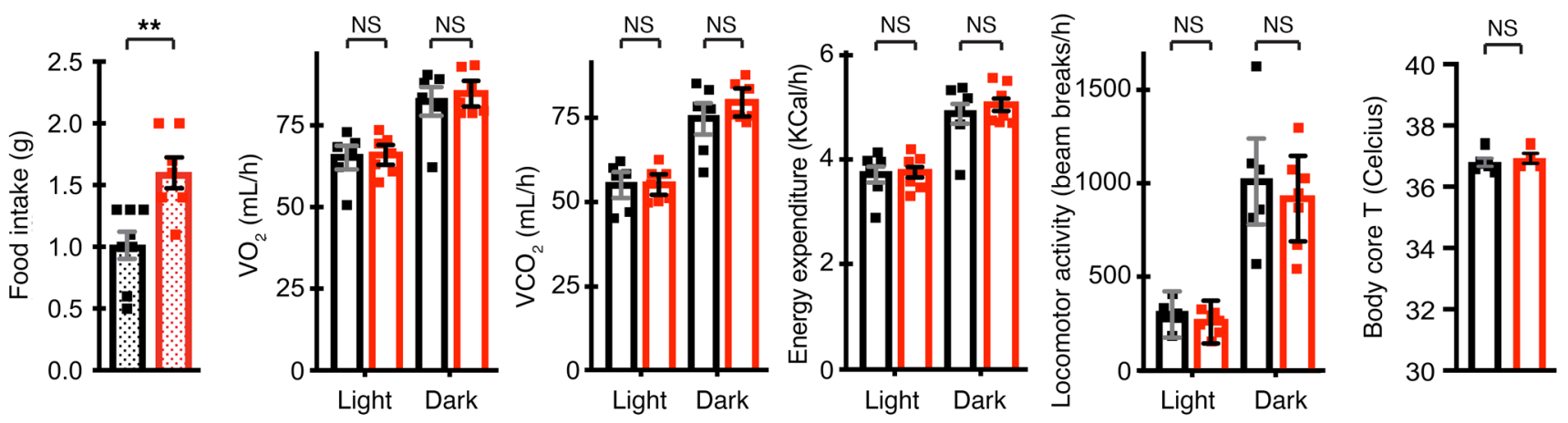

Figure 2. Short-term treatment with Flx induces a rapid increase in feeding. (A-D) WT female mice were treated with vehicle or Flx for 5 days. (A) Percentage of BW relative to BW on day 0 of treatment ( $n=16-17$ mice/group). (B) Percentage of WAT content relative to BW at the end of the treatment period ( $n$ $=16-20$ mice/group). (C) Cumulative food intake. (D) Daily percentage of food intake relative to that by vehicle-treated mice ( $n=14-16 \mathrm{mice} / \mathrm{group})$. (E) Food intake by WT female mice treated with vehicle or Flx for 14 hours during the active (dark) phase of the day ( $n=7-8$ mice/group). (F and G) WT female mice were treated with vehicle or Flx for 5 days. (F) Energy expenditure was measured by $\mathrm{VO}_{2}, \mathrm{VCO}_{2}$, heat production, and locomotor activity averaged over the last 4 days of the treatment ( $n=6-7$ females/group). (C) Core body temperature at the end of the treatment period ( $n=4-6$ mice/group). Values represent the mean \pm SEM. ${ }^{*} P \leq 0.05$, ${ }^{* *} P \leq 0.01$, and ${ }^{* *} P \leq 0.001$, by unpaired Student's $t$ test versus vehicle (A-D), ANCOVA (F, except for locomotor activity), or 2-way ANOVA followed by Šidák's test (locomotor activity in F). ${ }^{\dagger} P<0.001$, by paired Student's $t$ test for Flx compared with vehicle (E).

The short-term orexigenic effect of Flx is mediated by an autoinhibitory effect in serotonergic neurons of the dorsal raphe nuclei. To identify central sites where neuronal activity was acutely altered by Flx in an unbiased manner, we compared averaged maps of c-Fos ${ }^{+}\left(\right.$Fos $\left.^{+}\right)$, i.e., recently activated, neurons in intact whole brains of vehicle- and Flx-treated female mice using the tissue clearing/immunolabeling technique iDISCO+ paired with the cell detection and registration software ClearMap. A 3D automated comparison between $P$ value maps of c-Fos expression and the Allen Brain Atlas (ABA) annotation tool (2015 Allen Institute for Brain Science: brain-map.org/api/index.html) identified 3 known regulatory centers of feeding: the dorsal raphe nucleus (DRN) of the brainstem, the arcuate nucleus (ARC), and the paraventricular nucleus (PVN) of the hypothalamus as low Fos ${ }^{+}$areas in Flx- versus vehicle-treated mice (Figure 3, A and B, and Supplemental Figure $3, \mathrm{~A}$ and $\mathrm{B})$. We validated these results in coronal brain slices using conventional immunohistofluorescence (IHF) (Figure 3C). Next, to specify which subsets of neurons within these areas are affected by Flx, we assessed the colocalization between c-Fos and known neuronal markers associated with the regulation of feeding. In the DRN, IHF identified a significantly lower number of Fos $^{+} \mathrm{Tph}^{+}$neurons, a subset of neurons that negatively regulates feeding (19), in Flx- versus vehicle-treated female mice (Figure 3D and Supplemental Figure 3C), suggesting that Flx rapidly inhibited these serotonergic neurons. In pro-opiomelanocortin (POMC) neurons of the ARC, which drive satiety $(20,21)$, POMC levels and the number of $\mathrm{Fos}^{+} \mathrm{POMC}^{+}$neurons were decreased after Flx treatment compared with vehicle treatment (Figure 3E and Supplemental Figure 3E), suggesting that $\mathrm{Flx}$ also rapidly inhibited these neurons. Accordingly, the levels of $\alpha$-melanocyte-stimulating hormone $(\alpha-\mathrm{MSH})$, the POMC cleavage product that induces satiety by signaling in PVN (22), were also decreased in Flx- compared with vehicle-treated mice. This decrease manifested as early as 2 hours after Flx treatment but reached significance after 14 hours, reflecting the multistep posttranslational cleavage of POMC required for $\alpha-\mathrm{MSH}$ to be secreted (Figure 3F and Supplemental Figure 3, D and F). In contrast, the levels of NPY, another regulator of food intake (21), were not affected by the treatment with 
A
Annotation

Heatmap



Vehicle
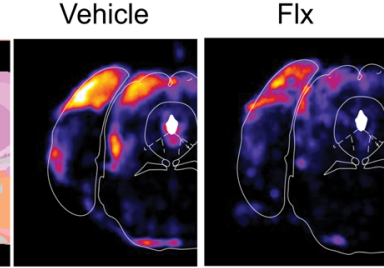

B
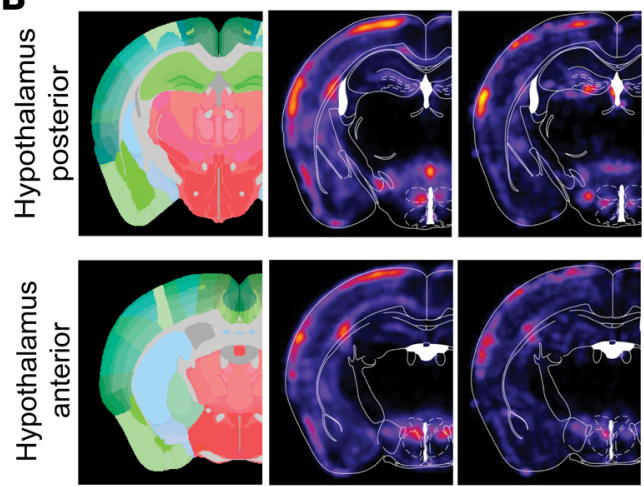

E

Vehicle

Flx
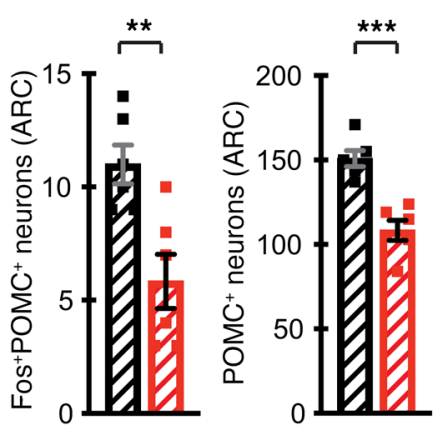
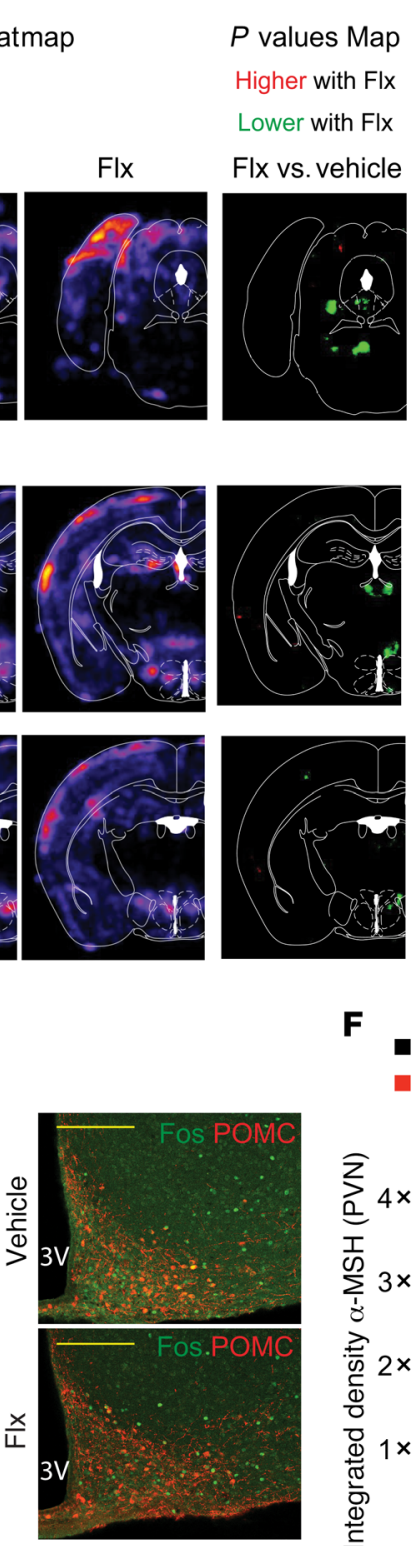

C
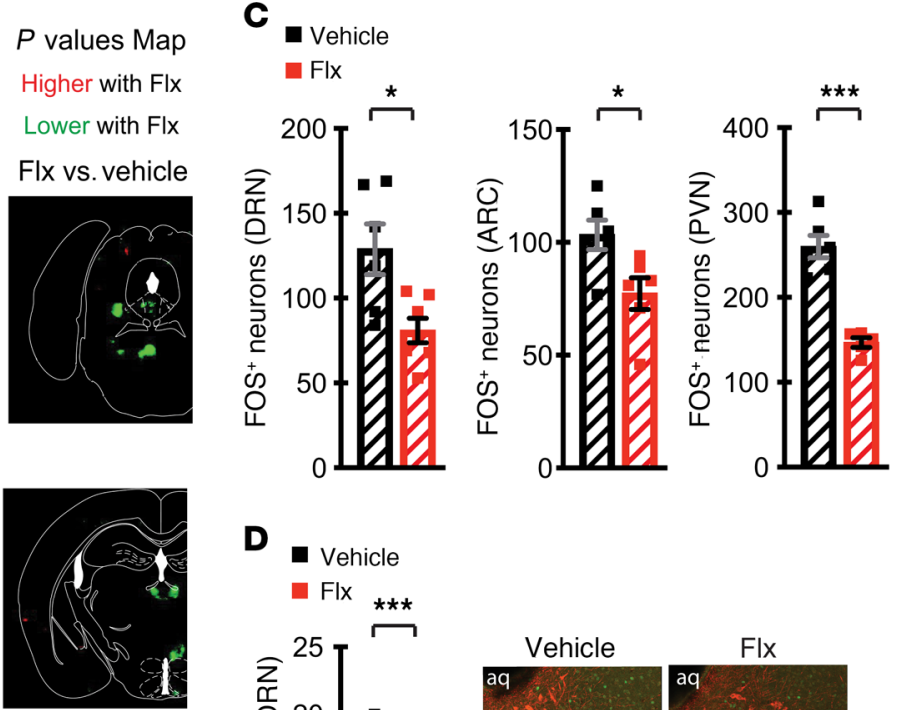

D vehicle
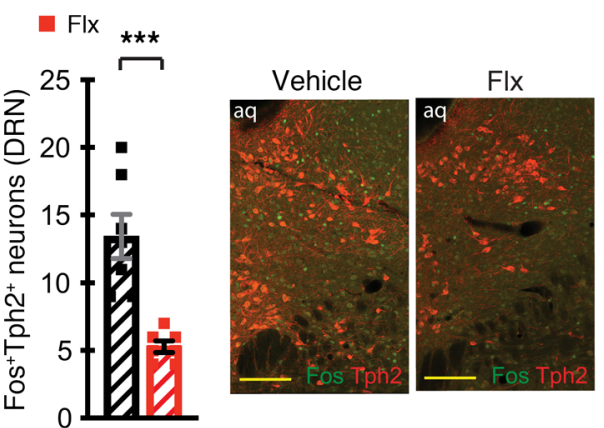

F

$\begin{array}{ll}\text { - Vehicle } & \mathrm{Fl} 14 \mathrm{~h}+1 \text {-day withdrawal } \\ \mathrm{Flx} & \mathrm{Fl} 14 \mathrm{~h}+2 \text {-day withdrawal }\end{array}$


Flx 14h Flx $14 \mathrm{~h}$

+1-day +2-day withdrawal withdrawal

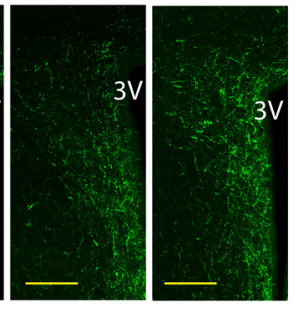

Figure 3. Acute treatment with FIx decreases the activity of DRN neurons and hypothalamic $\alpha$-MSH levels. (A and B) iDISCO+ whole-brain imaging and ClearMap analysis of c-Fos from compressed coronal views of the DRN (A) and hypothalamus (B) of WT female mice treated with vehicle (second column) or Flx (third column) for 2 hours ( $n=5-6$ mice/group). First column: ABA annotated image. Second and third columns: heatmaps. Fourth column: voxelbased statistical analysis. Regions with significantly different numbers of Fos ${ }^{+}$cells in the Flx versus vehicle treatment conditions are highlighted. (C) IHF validation of iDISCO+ results for brain slices from WT female mice treated with vehicle or Flx for 2 hours. Graphs show the quantification of Fos ${ }^{+}$neurons in the DRN, ARC, and PVN ( $n=5-7$ mice/group). ( $\mathbf{D}$ and $\mathbf{E}$ ) Representative images and quantification following double-IHF as indicated, in brain slices from WT female mice treated with Flx or vehicle for 2 hours (D, $n=7$ mice/group; E, $n=6$ mice/group). (F) Representative IHF images and levels of $\alpha$-MSH in PVN neurons quantified as integrated density in brain slices from WT female mice treated with vehicle or Flx for 14 hours. Flx was then withdrawn in some of the groups as indicated ( $n=12$ vehicle, $n=7$ mice/other groups). aq, aqueduct; $3 \mathrm{~V}$, third ventricle. Scale bars: $200 \mu \mathrm{m}$. Values represent the mean $\pm S E M$. ${ }^{*} P \leq$ $0.05,{ }^{* *} P \leq 0.01,{ }^{* *} P \leq 0.001$, and ${ }^{* * *} P \leq 0.0001$, by unpaired Student's $t$ test versus vehicle (C-E) or 1-way ANOVA followed by Dunnett's test (F).

Flx (Supplemental Figure 3G). To further confirm that Flx affects $\alpha$-MSH production, we treated a group of mice with Flx for 2 hours, followed by removal of the drug and IHF analysis of $\alpha$-MSH levels in the PVN 1 or 2 days later. As shown in Figure 3F, a 2-day withdrawal period normalized the density of $\alpha-\mathrm{MSH}$ signal. Altogether, these results identify serotonin-producing neurons in the DRN and the melanocortin circuitry of the hypothalamus as targets and putative mediators of the effects of Flx on feeding.
POMC neurons neither express Sert, the gene encoding the canonical target of Flx, nor secrete serotonin (refs. 19, 23; and the ABA annotation 2015 Allen Institute for Brain Science, brain-map. org/api/index.html). We therefore examined whether the shortterm orexigenic effect of Flx results from a serotonin-dependent cascade originating in the DRN that modulates the melanocortin system in the hypothalamus. We first assessed the effect of oral Flx on neuronal and feeding endpoints in mice lacking brain sero- 
tonin due to replacement of $T p h 2$, a gene encoding a rate-limiting enzyme in the central serotonin synthesis cascade, with the lac $Z$ reporter gene ( $\beta$-gal) (19). We observed no change in the number of $\mathrm{Fos}^{+} \beta$-gal ${ }^{+}$neurons in the DRN of Flx-treated mice compared with vehicle-treated Tph2-deficient mice, whereas the number of $\mathrm{Fos}^{+} \mathrm{Tph} 2^{+}$neurons in this area was decreased by Flx treatment in their control WT littermates (Figure 4A and Supplemental Figure 4, A and B). Moreover, Tph2-deficient mice treated with Flx exhibited no alteration in the number of $\mathrm{Fos}^{+} \mathrm{POMC}^{+}$neurons in the ARC and no decreased levels of $\alpha$-MSH in the PVN, but these effects were observed in their control WT littermates (Figure 4, B and C, and Supplemental Figure 4, C-E). These data suggest that brain serotonin could be required for the short-term effect of Flx on DRN and POMC neurons of the hypothalamus. Supporting the notion that the acute effect of oral Flx on feeding could be brain serotonin dependent, we observed no change in food intake or adiposity in Tph2-deficient mice after 5 days of Flx treatment, while both parameters were increased in their Flx-treated WT control littermates (Figure 4, D and E, and Supplemental Figure 4, F-I).

The therapeutic effect of Flx relies on an inhibition of serotonin reuptake by SERT that increases serotonin levels in the synaptic cleft (2). Thus, to test whether Flx acts on DRN neurons through this canonical action on SERT, we next analyzed mice lacking its encoding gene, Slc6a4 (24). Similar to what we observed in Tph2-deficient mice, the number of Fos ${ }^{+} \mathrm{Tph} 2^{+}$activated serotonergic neurons in the DRN was unchanged by the Flx treatment in SERT-deficient mice (Figure 4F and Supplemental Figure $4 \mathrm{~J}$ ). Taken together, these results indicate that an acute oral treatment with Flx increased feeding through a direct action on DRN serotonergic neurons and suggest that the changes in hypothalamic POMC and $\alpha$-MSH levels occurring in the hypothalamus were downstream of this effect.

DRN neurons send ascending projections to the ARC and the PVN, and serotonin signaling through the 5-hydroxytryptamine receptor 2c (Htr2c) in $\mathrm{POMC}^{+} \mathrm{ARC}$ neurons inhibits food intake $(5,9,25,26)$, an effect opposite to the rapid increase in feeding we observed with Flx treatment (Figure 2, C and D). DRN neurons express the inhibitory 5-hydroxytryptamine receptor 1a (Htr1a), and Htr1a negative regulation on the production of serotonin has been implicated in the delayed therapeutic efficacy of SSRIs on mood disorders $(27,28)$. We hypothesized that, by enhancing serotonin availability through SERT blockade, Flx could promote Htr1a negative feedback, thereby decreasing serotonin production and signaling in the downstream neurons that control feeding (29). If this hypothesis is correct, blocking Htrla function should prevent short-term Flx-induced hyperphagia as efficiently as enhancing the activity of Htr2c downstream of this effect. Indeed, coadministration of Flx and propranolol (Prop), which inhibits Htrla signaling (30), or Flx and lorcaserin (Lorca), a potent Htr2c agonist known to inhibit food intake $(9,31)$, were both sufficient to prevent increases in feeding, BW, and adiposity induced by Flx (Figure 4, G-N, and Supplemental Figure 4, K-N). These observations support the notion that a short-term oral Flx treatment increases food intake through serotonin-mediated autoinhibition of the Tph $2^{+} \mathrm{Htr}_{1} \mathrm{a}^{+}$DRN neurons that lowers serotonin production and therefore its signaling in $\mathrm{POMC}^{+} \mathrm{ARC}$ neurons to cause a decrease in $\alpha-\mathrm{MSH}$ production.
Impairment of $\mathrm{Htr} 2 \mathrm{c}$ expression, loss of STAT3 phosphorylation in ARC neurons, and low $\alpha$-MSH production explain the long-term orexigenic effect of Flx. To determine whether a similar mechanism causes the long-term orexigenic effect of Flx, we treated mice for 6 weeks with Flx or vehicle and analyzed the levels of $\alpha$-MSH by IHF. As seen with the 5-day treatment regimen, long-term exposure to Flx markedly decreased $\alpha$-MSH levels in the PVN, and withdrawal of the drug normalized $\alpha$-MSH levels after 4 weeks (Figure 5A). These data indicate that the effect of long-term Flx treatment on $\alpha-\mathrm{MSH}$ production is reversible. On the other hand, and in contrast to its preventative action on the acute effect of oral Flx on food intake, Lorca did not normalize feeding in mice pretreated for 5 weeks with Flx (Figure 5, B and C). Consequently, the mice treated long term with both Flx and Lorca gained weight and had an increase in fat mass comparable to that seen with controls treated with Flx alone (Figure 5, D-F, and Supplemental Figure 5, $\mathrm{A}$ and $\mathrm{B})$. The difference in the impact of Lorca on the short-term and long-term orexigenic activity of Flx suggests that Htr2c signaling might have been impaired and/or that a negative effect on another regulator of $\alpha$-MSH production was occurring.

We have previously demonstrated that long-term treatment with Flx decreases levels of Htr2c and its activation and phosphorylation of cAMP response element-binding protein (CREB), its downstream effector in the hypothalamic neurons regulating bone remodeling $(32,33)$. Since this receptor also mediates the effect of serotonin on feeding (34-36), we assessed whether the long-term effect of Flx is associated with a decrease in Htr2c levels in ARC neurons. Double-IHF staining confirmed a weakened Htr2c signal in $\mathrm{POMC}^{+} \mathrm{ARC}$ neurons in mice treated for 6 weeks with Flx compared with those treated with vehicle (Figure 5G, arrows). POMC levels were also decreased in the ARC region (Figure $5 \mathrm{H}$ ). Given that $P O M C$ is also expressed in Htr2c-negative neurons of the ARC (36) and that phosphorylated STAT3 (p-STAT3) is a major regulator of its expression (37), we next tested whether Flx affects this transcription factor. We found that p-STAT3 levels were decreased in ARC neurons of mice treated for 6 weeks with Flx (Figure 5I). Hence, upon long-term use, Flx decreased $\alpha$-MSH production by interfering with 2 pathways regulating POMC expression: in 1 subset of neurons it lowered Htr2c expression and impaired the CREB-dependent activation of the POMC promoter (32), and in other neurons, it decreased its STAT3-dependent induction by hampering the phosphorylation of this factor.

Flx impairs STAT3 phosphorylation, CREB activation, and $P O M C$ expression in human neuronal cells. To begin evaluating the potential relevance of this dual inhibitory mechanism to human biology, we analyzed the effect of Flx on the human neuroblastoma cell line SH-SY5Y, which, upon treatment with all-trans retinoic acid (ATRA), expressed mature neuronal markers (Supplemental Figure 6A). Even though POMC expression was low in these cells, it was decreased by a 2-hour treatment with Flx (Figure 6A). Likewise, Western blot analysis respectively identified a $20 \%$ and $15 \%$ decrease in the p-STAT3/STAT3 and p-CREB/ CREB ratios in Flx-treated cells (Figure 6, B and C). This effect was observed in all experiments and was specific to the p-STAT (Tyr705) form, which is the main form regulating POMC expression, as STAT3 ERK-dependent Ser727 phosphorylation was not affected (Supplemental Figure 6B). 
A

O Tph2-//vehicle

O Tph $2^{-/ F \mid \mathrm{F}}$

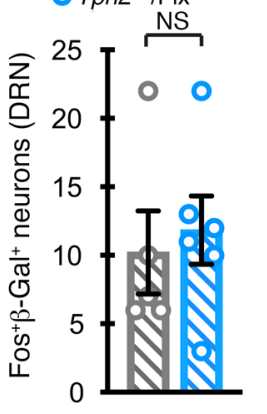

D

$\rightarrow-T p h 2^{-1 / v e h i c l e ~}$

- - Tph $2^{-1 / F I x}$



G
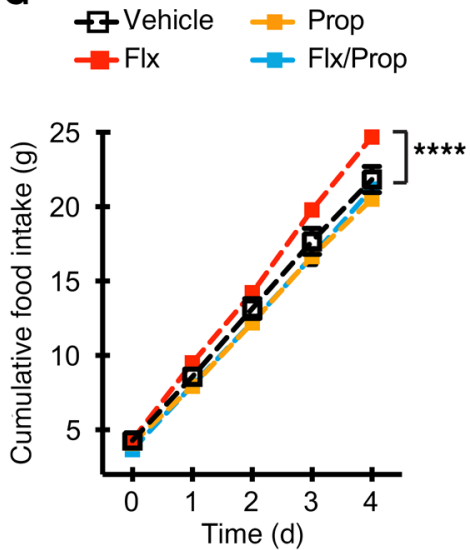

$\mathbf{K}$
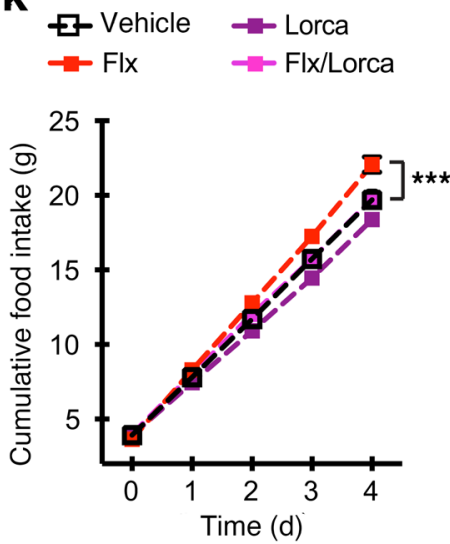

C

C O Tph $2^{-}$-vehicle

O Tph2--IFIx
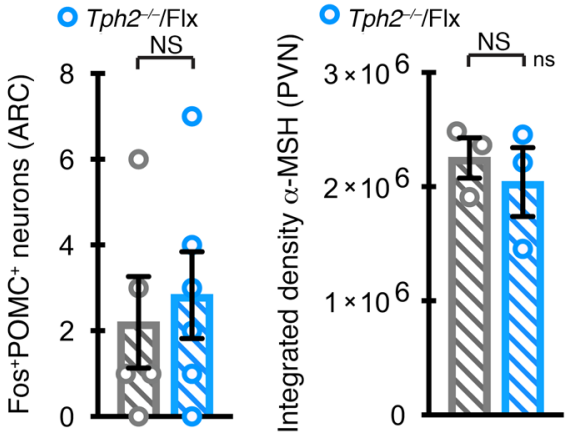

E

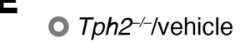

$\mathbf{F}$

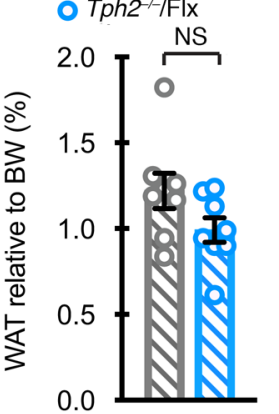

(5)

O Sert'/FIx



Figure 4. Short-term Flx-induced hyperphagia is serotonin dependent and can be countered by pharmacological inhibition of Htr1a or activation of Htr2c signaling.

(A and B) IHF in brain slices from $T p h 2^{-/-}$female mice treated with Flx or vehicle for 2 hours. (A) Quantification of $\mathrm{Fos}^{+} \beta$-gal ${ }^{+}$neurons in the DRN or (B) Fos ${ }^{+} \mathrm{POMC}^{+}$ neurons in the ARC ( $n=5-6$ mice/group). (C) IHF in brain slices from $\mathrm{Tph}^{-/-}$female mice treated with Flx or vehicle for 14 hours. Graph shows the levels of $\alpha-M S H$ measured as integrated density ( $n=3$ mice/group). ( $D$ and E) Tph ${ }^{-/-}$ females were treated with Flx or vehicle for 5 days $(n=8$ mice/group). (D) Cumulative food intake. (E) Percentage of WAT content relative to BW. (F) Quantification of Fos ${ }^{+} \mathrm{Tph}^{+}$neurons in the DRN by IHF in brain slices from $\mathrm{Sert}^{-1-}$ female mice treated with Flx or vehicle for 2 hours ( $n=3-5$ mice/group). (G-J) WT females were treated for 4 days with vehicle, Flx, Prop, or both drugs ( $n=7-8$ mice) group). (C) Cumulative food intake. (H) Percentage of BW relative to day 0 of treatment. (I) Percentage of WAT content relative to BW. (J) Fat content measured by EchoMRI relative to BW. (K-N) WT female mice were treated for 4 days with vehicle, Flx, Lorca, or both drugs ( $n=7-8$ mice/group). (K) Cumulative food intake. (L) Percentage of BW relative to day 0 of treatment. (M) Percentage of WAT content relative to BW. (N) Fat content measured by EchoMRI relative to BW. Values represent the mean \pm SEM. ${ }^{*} P \leq 0.05,{ }^{* *} P \leq 0.01,{ }^{* *} P \leq 0.001$, and ${ }^{* * * *} P \leq$ 0.0001 , by unpaired (A-C, E, and F) or paired (D) Student's $t$ test versus vehicle, or by 2 -way ANOVA (G and $\mathbf{K}$ ) or 1-way ANOVA (H-J and $\mathbf{L}-\mathbf{N}$ ) followed by Tukey's test.

H

$\begin{array}{ll}\text { - Vehicle } & \text { Prop } \\ \text { - Flx } & \text { Flx/Prop }\end{array}$


$\mathbf{L}$

- Vehicle - Lorca

- Flx a Flx/Lorca
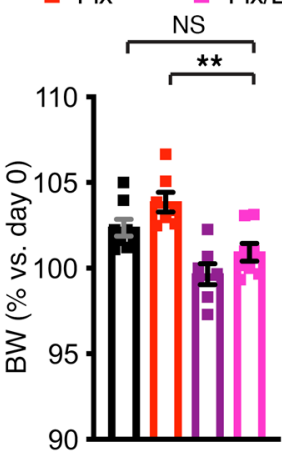

I

$\mathbf{J}$
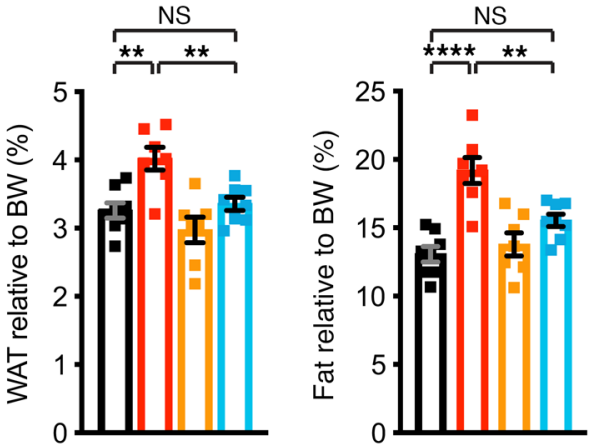

M

$\mathbf{N}$
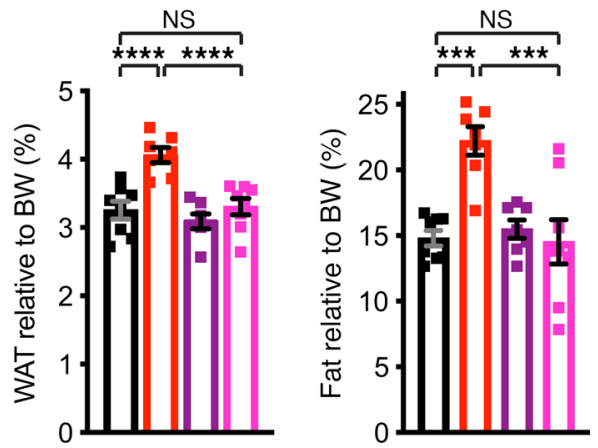

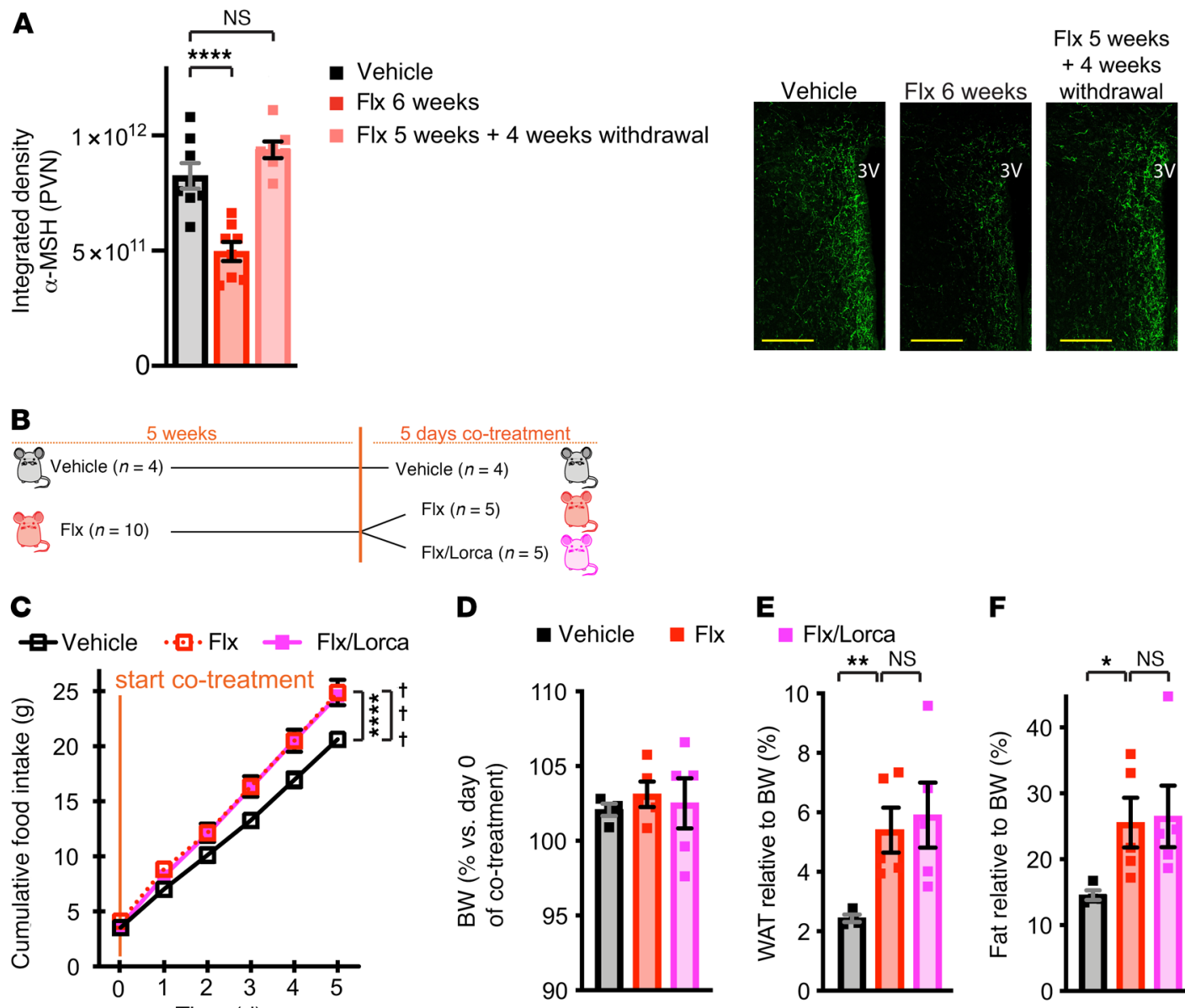

E
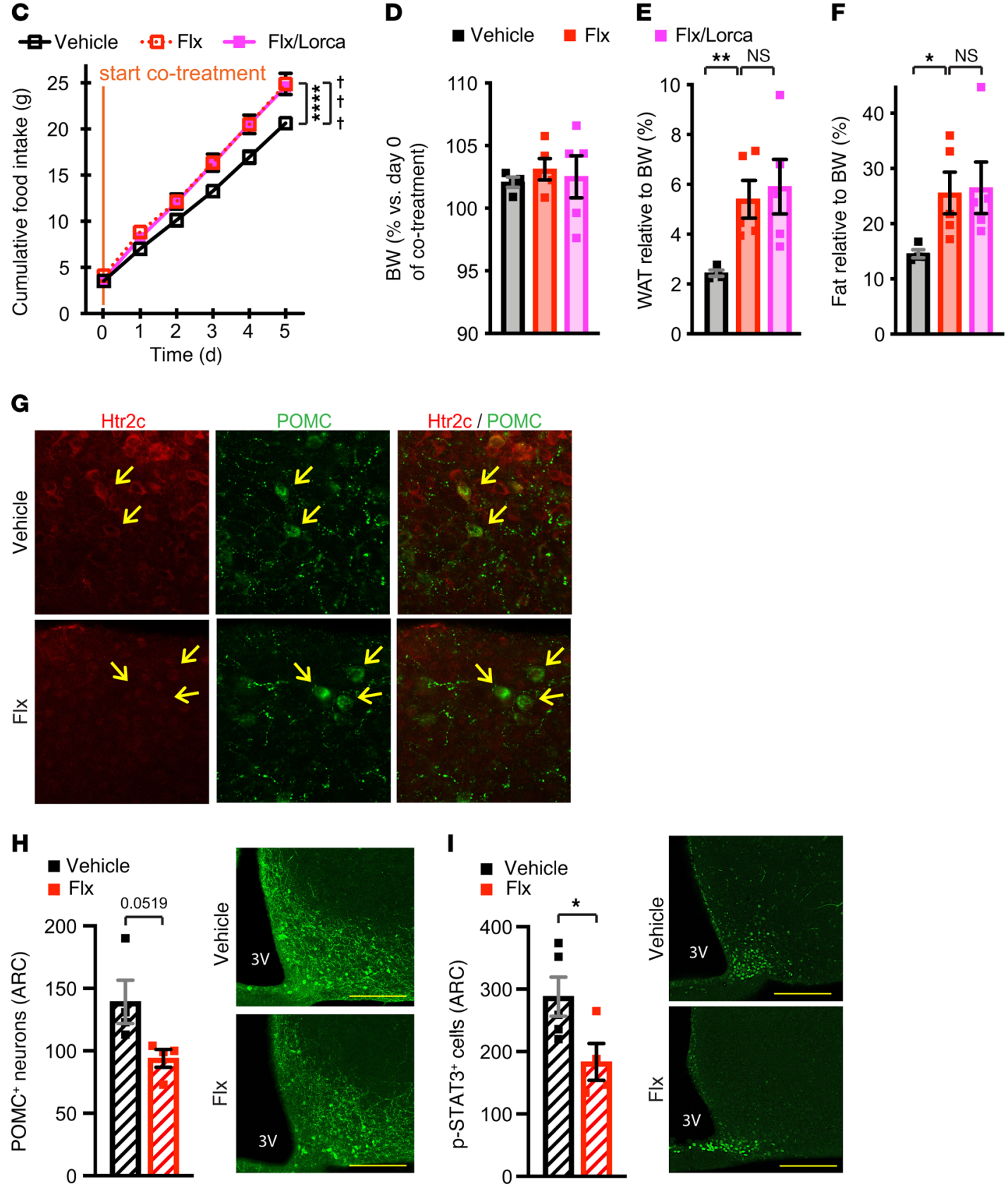
Figure 5. Long-term treatment with Flx impairs Htr2c signaling, STAT3 phosphorylation, and $\alpha$-MSH production in hypothalamic neurons. (A) Representative images of IHF and levels of $\alpha$-MSH quantified as integrated density in brain slices from WT female mice treated with Flx or vehicle for 6 weeks. Flx was then withdrawn in some of the groups as indicated $(n=7-8$ mice/group). (B-F) WT female mice were treated for 5 weeks with vehicle or Flx and were then treated with vehicle or Flx alone or Flx and Lorca for 5 additional days ( $n=4-5$ mice/group). (B) Schema of experimental design. (C) Cumulative food intake. (D) Percentage of BW relative to day 0 of cotreatment. (E) Percentage of WAT content relative to BW. (F) Fat content measured by EchoMRI relative to BW. (G-I) WT females were treated with vehicle or Flx for 6 weeks ( $n=4$ mice/group). (C) Representative IHF images of Htr2c expression in $\mathrm{POMC}^{+} \mathrm{ARC}$ neurons. Arrows point to $\mathrm{POMC}^{+}$cells. Representative images and quantification of (H) POMC ${ }^{+}$and (I) p-STAT3 ${ }^{+}$ neurons in arcuate nuclei. Scale bars: $200 \mu \mathrm{m}$. Values represent the mean \pm SEM. ${ }^{*} P \leq 0.05$ and ${ }^{* *} P \leq 0.01$, and ${ }^{* * *} P \leq 0.0001$, for vehicle versus FlX treatment; ${ }^{\dagger+t} P \leq 0.001$, for vehicle versus Flx plus Lorca treatment. Significance was determined by 1-way (A, E, and F) or 2-way (C) ANOVA followed by Tukey's test, or by unpaired Student's $t$ test (H and I).

Newer-generation SSRIs are also associated with weight gain following long-term use (14). We therefore tested whether several of these compounds were inhibiting STAT3 and/or CREB phosphorylation. As shown in Figure 6D, all 4 compounds tested decreased CREB phosphorylation in differentiated SY5Y cells, although the effect with fluvoxamine did not reach statistical significance. Sertraline, escitalopram, and fluvoxamine also inhibited STAT3 phosphorylation to the same extent as Flx (Figure 6E). Of note, paroxetine, which does not elicit a significant weight gain in patients (14), did not have a negative effect on this process (Figure 6E).

Pharmacological enhancement of Mc4R activity compensates both short- and long-term anorexigenic effects of Flx and blocks weight gain. The results presented above indicate that a decrease in $\alpha$-MSH signaling in the PVN was ultimately responsible for the paradoxical orexigenic effect of oral Flx. Since $\alpha$-MSH suppresses hunger by signaling through the melanocortin 4 receptor (Mc4r) expressed on PVN neurons (22), we reasoned that exogenous stimulation of Mc4r signaling could block this orexigenic effect. Such treatment should be able to compensate for the decreased $\alpha$-MSH production caused by both the short-term firing inhibition of $\mathrm{DRN}^{\mathrm{Tph} 2}$ neurons by Htr1a negative feedback and the decrease in hypothalamic Htr2c signaling and CREB/ STAT3 activity associated with long-term Flx treatment. To test this hypothesis, we injected mice with lipocalin 2 (Lcn2), a recently described anorexigenic hormone that signals directly through $\mathrm{Mc} 4 \mathrm{r}$ in the hypothalamus (38), either during a 5-day cotreatment or for 5 days after 5 -week pretreatment with Flx (Figure 7A). We observed that, in both cases, Lcn 2 treatment decreased food intake in Flx-treated mice compared with mice treated with Flx alone (Figure 7, B and F). Accordingly, BW and adiposity were also normalized in mice cotreated with Flx and Lcn 2 compared with Flx-treated ones (Figure 7, C-E and G-I, and Supplemental Figure 7, A-E). Importantly, this treatment with Lcn 2 did not interfere with the beneficial effect of Flx on mouse behavior, as assayed by the classic marble-burying test measuring anxiety (Figure 7J). Thus, increasing Mc4r signaling in the hypothalamus by peripheral injection of Lcn 2 could compensate for the decrease in $\alpha$-MSH signaling induced by Flx and thereby block its short-term as well as long-term orexigenic effects without affecting its therapeutic benefit.

\section{Discussion}

We show here that long-term oral treatment with Flx promotes weight gain in healthy mice by increasing food intake. This effect is centrally mediated and impinges on the positive regulation of the melanocortin pathway by brain serotonin through 2 different mechanisms (Figure 8). Flx initially triggers an Htr1a autoinhibition of $\mathrm{Tph} 2^{+} \mathrm{DRN}$ neurons of the brainstem that decreases their positive action on $\mathrm{POMC}^{+}$ARC neurons. Pharmacologically blocking Htr1a or compensating for the decrease of serotonin signaling by direct stimulation of Htr2c with Lorca can block this initial orexigenic effect of Flx. When the Flx treatment period is extended, a decrease in hypothalamic Htr2c levels and signaling, combined with a negative effect of Flx in ARC neurons on the phosphorylation of CREB and STAT3, two major regulators of POMC expression, induces a decrease in $\alpha-\mathrm{MSH}$ levels, an increase in food intake, and weight gain. In this second phase, Lorca has no effect, whereas acting downstream of Htr2c and POMC neurons by stimulating Mc4r signaling with Lcn2, can block the orexigenic effect of Flx and hinder weight gain. Our data also show that Flx and other SSRIs could inhibit STAT3 and CREB phosphorylation, and thereby $P O M C$ expression, in human neuronal cells. Although preliminary, this observation suggests that a similar mechanism could affect feeding in mice and humans and, therefore, that MC4R agonists could be used to control SSRIinduced weight gain in patients.

Our observations explain how Flx impinges on known components of the serotonin/melanocortin pathway to reverse its effect on feeding. For instance, the initial Htr1a-mediated inhibition of Tph $2^{+}$DRN neuron activity and its lowering effect on downstream serotonin signaling has been previously described, although in the context of mood and anxiety, not of food intake (27). This negative feedback inhibition in response to increased intersynaptic serotonin levels in the DRN is believed to account for the delayed therapeutic response to SSRIs; these drugs would be able to achieve their functional effect only when desensitization of Htr1a has occurred (27). Such a latter mechanism, however, does not explain the weight loss observed within 24 hours of treatment in studies performed in rats $(6,7)$. A major difference between these and our studies relates to the route of Flx administration. We opted for an oral treatment that would more precisely mimic the conditions of administration in patients, whereas these early studies delivered Flx via intraperitoneal injections. Whether the divergence of the effects relates to differences in processing, bioavailability, or kinetics of brain concentration remains to be determined. Notably, our results are consistent with previous observations reported in studies in which Flx was delivered orally, although those studies used different doses and were not designed to evaluate this drug's effect on feeding $(39,40)$.

Although consistent with the BW gain reported by patients on long-term SSRI treatment, the orexigenic effect of Flx we observed after 6 weeks of treatment conflicted with the effect expected from a drug acting by increasing brain serotonin signaling. This apparent contradiction is explained by the combination of multiple molecular events taking place downstream of serotonin sig- 
A
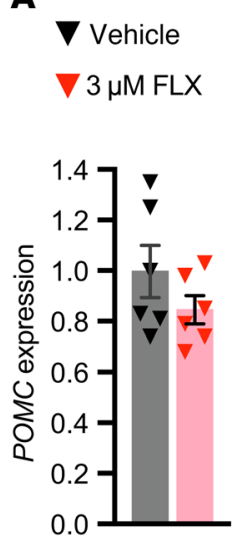

B

Vehicle

$\nabla 3 \mu \mathrm{M} F \mathrm{FL}$

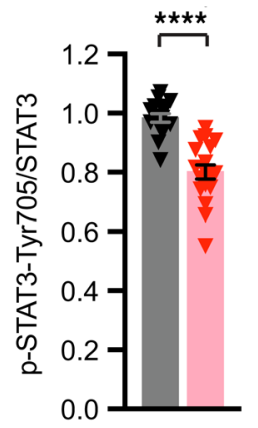

C

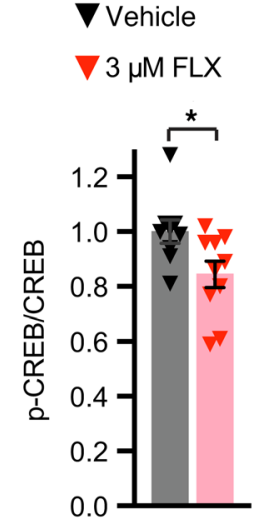

D

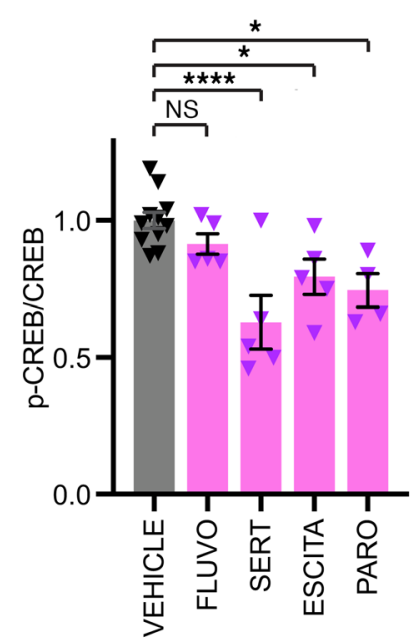

E

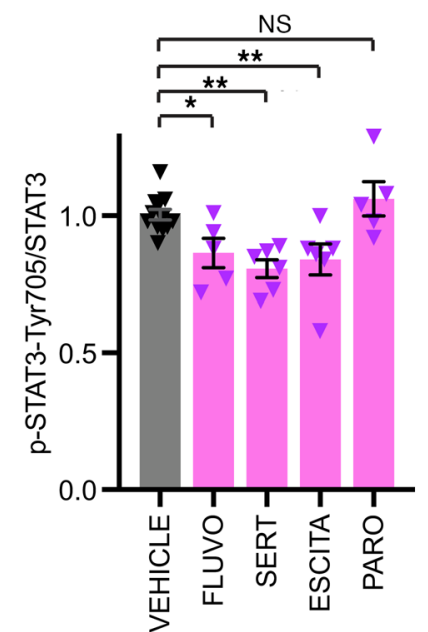

Figure 6. FIX and other SSRIs interfere with STAT3 and CREB phosphorylation in human neuronal cells. SY5Y neuron-like cells were differentiated for 5 days and treated with vehicle, Flx, or other SSRIs as indicated. (A) Gene expression analysis of POMC by qPCR after 4 hours of treatment. (B and E) Analysis of p-STAT3 and (C and D) p-CREB by Western blotting after a 2-hour treatment with Flx or vehicle. FLUVO, fluvoxamine; SERT, sertraline; ESCITA, escitalopram; PARO, paroxetine. Values represent the mean \pm SEM. ${ }^{*} P \leq 0.05,{ }^{* *} P \leq 0.01$, and ${ }^{* * *} P \leq 0.0001$, by Student's $t$ test versus vehicle (B and C) or 1-way ANOVA followed by Dunnett's test ( $\mathbf{D}$ and $\mathbf{E}$ ).

naling in ARC neurons that our study and previous reports uncovered. Indeed, the fact that increased intersynaptic serotonin levels are expected to trigger Htr2c desensitization (41), combined with the observation that Flx decreases Htr2c levels in hypothalamic neurons (ref. 32 and Figure 5G), should dampen serotonin signaling and therefore blunt the potential effect of the SSRI-mediated increase in serotonin levels. The inhibition of the phosphorylation of STAT3 and CREB would add to this loss of Htr2c signaling and concur to prompt the decrease in $\alpha$-MSH levels we observed. The fact that the Htr2c agonist Lorca can block Flx-induced, serotonin-dependent initial hyperphagia and weight gain but is ineffective in doing so upon long-term pretreatment is consistent with this multilevel block in the Htr2c-mediated regulation of feeding.

Our in vitro experiments using differentiated SY5Y-derived neuronal cells suggest that Flx can inhibit POMC expression through its negative effect on p-STAT3 and p-CREB in human cells, as it does in mouse neurons. Although these data cannot be interpreted to model the situation in humans, if they could be verified in patients treated with Flx, it would mean that Lorca or other compounds that enhance serotonin production or Htr2c signaling may not be successful in blocking such an effect in patients on long-term SSRI treatment. Targeting instead the pathway downstream of these factors may a better therapeutic strategy. Specifically, direct stimulation of the MC4R by injecting the satiety hormone LCN2 (38), whose anorexigenic effect is conserved between rodents, nonhuman primates, and humans (42), could block the effect of Flx on feeding and therefore limit weight gain in patients. Additional studies analyzing the pharmacology and safety profile of LCN2 are, however, needed to establish its value as a co-drug and, in particular, to assess its effect on blood pressure and heart rate, as other MC4R agonists have been clinically abandoned because of unacceptable increases in these parameters (43). In that respect, cotreatment with SSRIs and the MC4R selective agonist setmelanotide may offer a better option, since this drug did not show adverse cardiovascular effects while significantly reducing weight loss and hunger scores in phase III clinical trials in individuals with POMC or leptin receptor (LEPR) deficiency $(44,45)$.

\section{Methods}

Study design. The study was performed using mouse models and a human neuron-like cell line. In vivo, group sizes were determined by performing a power calculation to lead to an $80 \%$ chance of detecting a significant difference $(P \leq 0.05)$. All experiments were laboratory controlled and were designed with specific final endpoints, which were arbitrarily designed to represent acute, short-term, and long-term treatments. In all experiments, the animals were randomly assigned to the specific treatment groups. Any mouse displaying immobility, huddled posture, an inability to eat or drink, ruffled fur, self-mutilation, vocalization, wound dehiscence, or signs of hypothermia was excluded from the study.

All values represent biological replicates and are indicated by group size $(n)$ in the figure legends or with dots in the bar graphs. For in vivo data, each $n$ value corresponds to a single mouse. For in the vitro data, each $n$ value corresponds to a replicate; 2-6 independent experiments were performed with triplicates for each condition. BW measurements and feeding experiments for WT mice were performed several times, except for in the 12-week treatment experiment.

Animals and in vivo treatments. WT and Sert ${ }^{-1}$ mice were purchased from The Jackson Laboratory. The generation of $T p h 2^{-/}$mice was reported previously (19). All mice analyzed were on a C57Bl6/J background. Virgin female and male mice, aged 3-6 months, were used for analysis, depending on the length of treatment.

Flx hydrochloride USP ( $20 \mathrm{mg} / \mathrm{kg} /$ day; Combi-Blocks) was added aseptically to the drinking water, and bottles were replaced every other day. Prop ( $0.5 \mathrm{mg} /$ day; MilliporeSigma) was added aseptically to the drinking water. Recombinant Lcn2 (200 ng/g/day in PBS) was prepared as previously described (38) or Lorca hydrochloride $(3 \mathrm{mg} / \mathrm{kg} /$ day) in PBS (AbMole BioScience) was injected intraperitoneally daily 
A

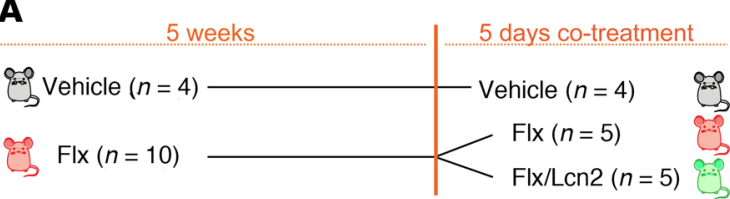

B
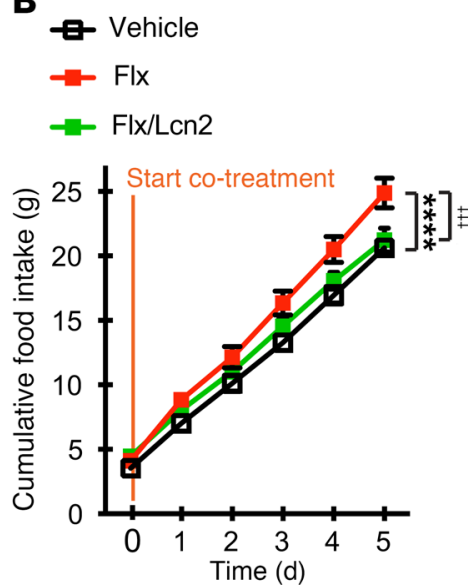

F


c

- Vehicle

- Flx

Flx/Lcn2
D

- Vehicle
- Flx

- Flx/Len2
E

- Vehicle

- $\mathrm{Flx}$

- Flx/Lcn2
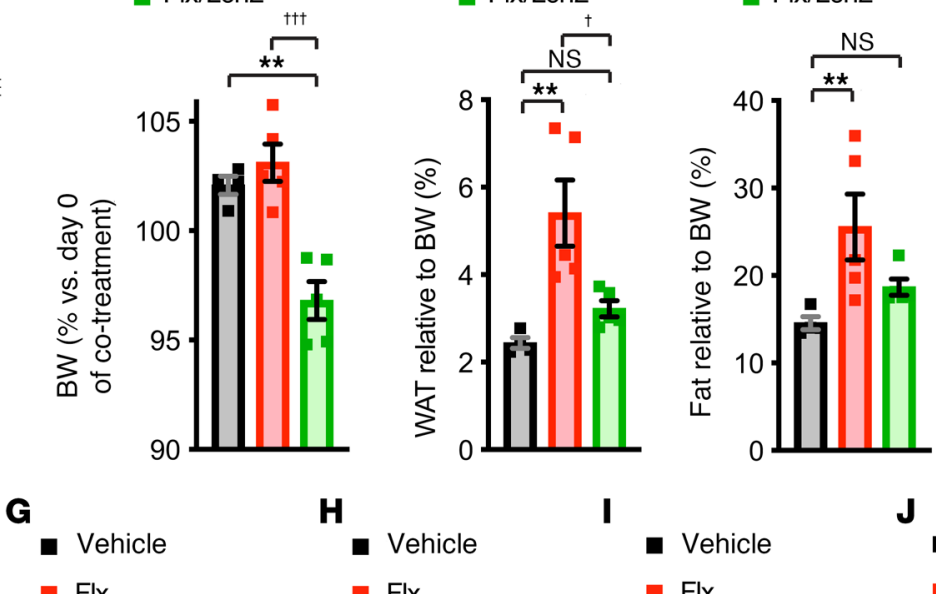

H



I
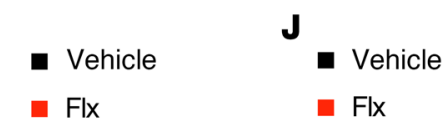

Flx

- Lcn2

- Lcn2

- Flx/Lcn2

- Lcn2

Lcn2

- Flx/Lcn2

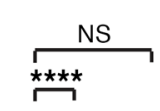

- Flx/Lcn2

- Flx/Lcn2
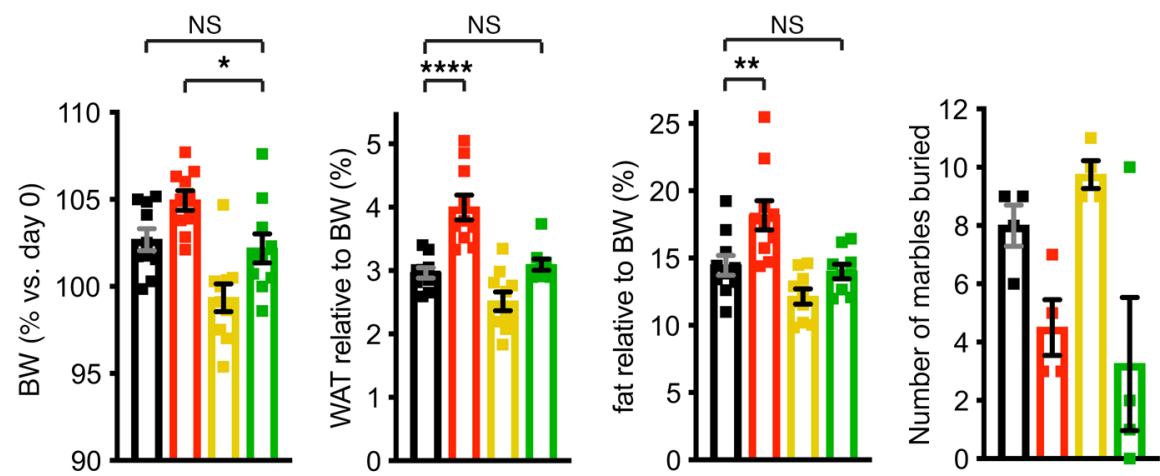

Figure 7. Cotreatment with Len2 can block the long-term effects of Flx on feeding and BW. (A-E) WT female mice were treated for 5 weeks with vehicle or Flx and then with vehicle, Flx, or Flx plus Len2 for 5 additional days ( $n=4-5$ mice/group). (A) Schema of the experimental design. (B) Cumulative food intake. (C) Percentage of BW on the last day of cotreatment relative BW on day 0 of cotreatment. (D) Percentage of WAT content relative to BW. (E) Fat content relative to BW. (F-J) WT female mice were treated for 4 days with vehicle, Flx, Lcn2, or both drugs as indicated ( $n=10$ mice/group). (F) Cumulative food intake. (G) Percentage of BW on the last day of treatment relative to BW on day 0. (H) Percentage of WAT content relative to BW. (I) Fat content relative to BW. (J) Marble-burying test. Values represent the mean $\pm \mathrm{SEM}$. ${ }^{*} P \leq 0.05,{ }^{*} P \leq 0.01,{ }^{* * *} P \leq 0.001$, and ${ }^{* * * *} P \leq 0.0001$, for vehicle versus Flx; ${ }^{\dagger} P \leq 0.05$ and ${ }^{+\dagger+} P \leq 0.001$, for Flx compared with Flx ${ }^{+}$Lcn2 ${ }^{+}$. Two-way ANOVA (B and $\mathbf{F}$ ) or 1-way ANOVA (C-E and G-J) followed by Tukey's test.

at 6:45 pm. Vehicle control mice were injected with a similar volume of PBS. Treatments represented in Figure 4, B-F, and Figure 6, A-D, were done simultaneously using mice from the same vehicle-treated and Flx-treated groups.

Physiological measurements. For BW measurements over a 6-week period, animals were housed in home cages by groups of 5 with ad libitum access to water and chow. Measurements were taken every week between 9:00 am and 10:00 am.

For BW and food intake over 4 or 5 days, animals were kept individually in home cages with ad libitum access to water and chow. After 4 days of acclimation, basal BW was measured daily, between
9:00 am and 10:00 am, and food was weighed daily at 9:00 am and $6: 45 \mathrm{pm}$, for 4 or 5 additional days. Mice were then treated with water supplemented with Flx, or water supplemented with Flx and Prop (or were injected with Lcn2, Lorca, or vehicle). At the beginning of the treatment, chow was replaced with fresh chow (150-170 $\mathrm{g}$ ), and BW and food consumption were measured as above. The BW percentage was represented relative to the average of the last $3 \mathrm{BW}$ measurements prior to treatment.

For food intake measurements over a 14-hour period, animals were placed individually in home cages with ad libitum access to water and chow. After 4 days of acclimation, the mice were weighed to 

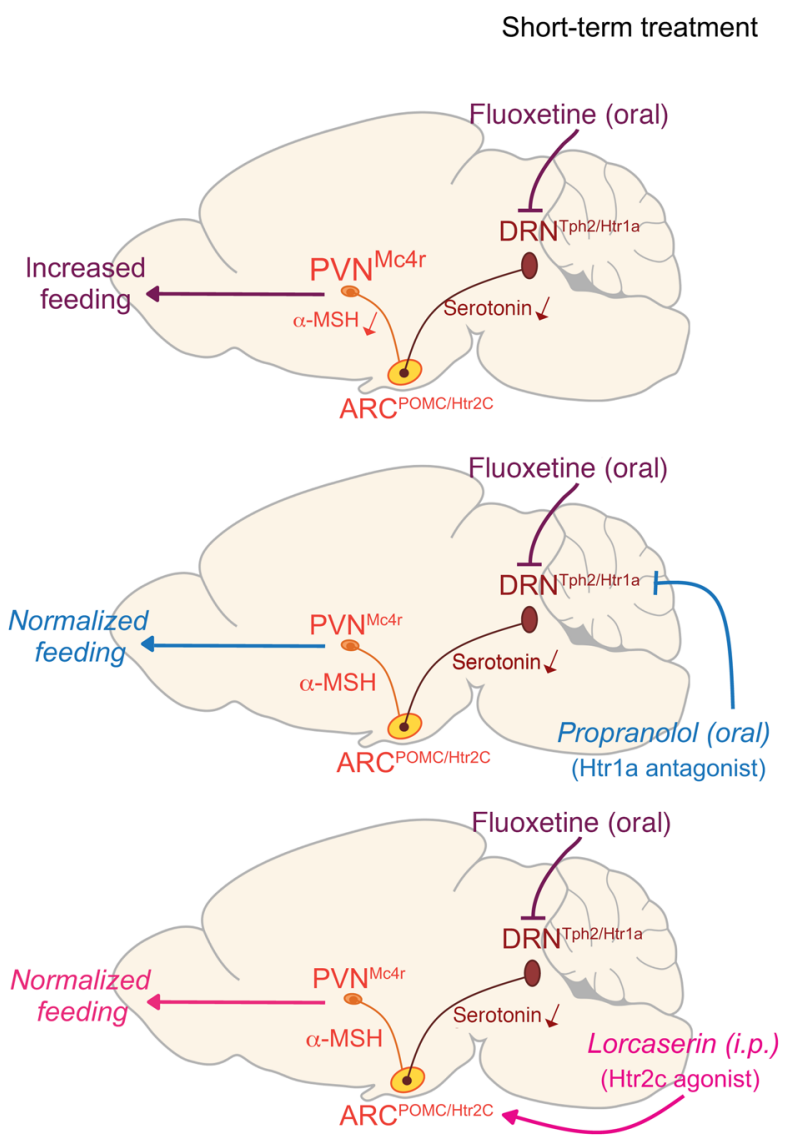

Long-term treatment
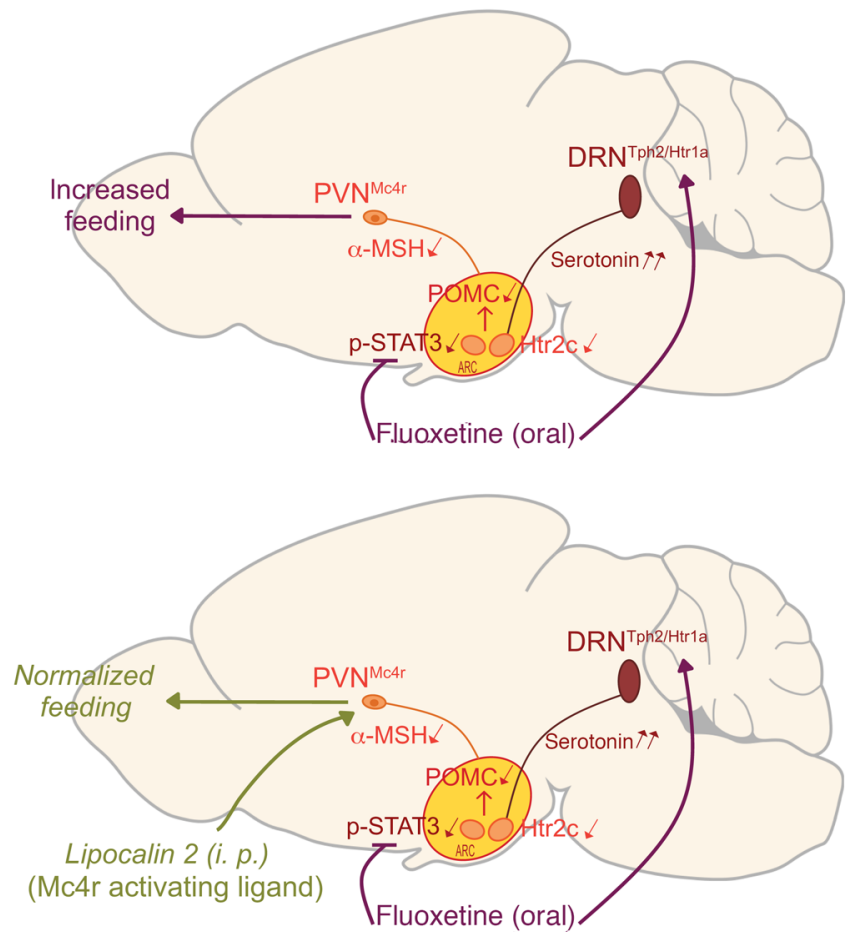

Figure 8. Mechanisms of Flx-induced short-term and long-term hyperphagia and weight gain. In the short term (left), within hours of oral treatment with Flx, Tph2 $2^{+} \mathrm{Htr1a}^{+}$DRN neurons are inhibited, leading to a decrease in the serotonin-dependent activation of POMC ${ }^{+} \mathrm{Htr} 2 \mathrm{C}^{+} \mathrm{ARC}\left(\mathrm{ARC} \mathrm{POMC}^{\mathrm{Htr} 2 \mathrm{C}}\right.$ ) and

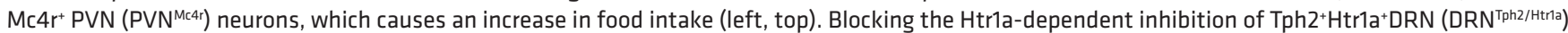
neurons using Prop (left, middle) or activating POMC ${ }^{+} \mathrm{Htr}_{2} \mathrm{c}^{+} \mathrm{ARC}\left(\mathrm{ARC} \mathrm{POMC} / \mathrm{Htr2c}^{\mathrm{C}}\right.$ ) neurons using Lorca (left, bottom) can therefore normalize feeding in this setting. Upon long-term treatment (right), however, Flx decreases Htr2c expression and signaling and inhibits STAT3 phosphorylation in ARC neurons, resulting in reduced $\alpha$-MSH production (right, top). This noncanonical and multifactorial activity of Flx explains the paradoxical hyperphagia and weight gain associated with its long-term use as well as the failure of Lorca to counter this effect. In contrast, cotreatment with Lcn2, a Mc4r ligand, can normalize feeding and prevent weight gain (right, bottom).

ensure that no difference in BW existed between the groups (Supplemental Figure 8, A and B). The mice were then treated with vehicle or Flx and water at 6:45 pm, and the chow was replaced with fresh chow (150-170 g) and food weighed at 9:00 am.

Energy expenditure was assessed through measurement by indirect calorimetry of oxygen consumption $\left(\mathrm{VO}_{2}\right)$, carbon dioxide production $\left(\mathrm{VCO}_{2}\right)$, heat production, and locomotor activity using an automated home cage phenotyping system (TSE Systems), except for the experiments in Supplemental Figure 2, L and O, for which a 6-chamber Oxymax system (Columbus Instruments) was used. Mice were singled housed in a climate-controlled chamber (temperature: $22^{\circ} \mathrm{C}$; humidity: 55\%; 12-hour light/12-hour dark cycle) with ad libitum access to water and chow. After 48 hours (Oxymax, Columbus Instruments) or 7 days (TSE Systems) of adaptation to social isolation, the mice were treated with vehicle or Flx for 5 days (for Figure 1C, the mice were treated with vehicle or Flx for a period of 6 weeks, including adaptation to social isolation and the recording time). Data were collected and analyzed as recommended by the manufacturers. Day-by-day values or averaged measurements of the last 4 days are represented as indicated. Locomotor activity was recorded as beam breaks converted into distance/velocity, measuring activity in 3D. Beam breaks by mice were analyzed in the metabolic cage using custom software. The respiratory exchange ratio (RER) and energy expenditure were calculated from $\mathrm{VO}_{2}$ and $\mathrm{VCO}_{2}$ production data. In the TSE System, measurements from days 1-3 and 4-6 were used for energy expenditure assessments.

Fat content and body temperature were measured using echo magnetic resonance imaging (EchoMRI). Gonadal and subcutaneous white adipose tissue (WAT) was dissected and weighed at the time of euthanasia and represented relative to BW.

iDISCO+ whole-brain clearing, labeling, imaging, and ClearMap analysis. Female mice were placed individually in home cages with water and chow ad libitum. After 4 days of acclimation, they were fasted overnight. In the morning (9:00 am), food was added and water was replaced with water supplemented with vehicle or water supplemented with Flx. Mice were euthanized after 2 hours and transcardially perfused with $\mathrm{PBS}$ followed by $4 \%$ paraformaldehyde (PFA). Brains were postfixed for 24 hours, after which immunolabeling with rabbit c-Fos, 1:4000 (Synaptic Systems, catalog 226 003, RRID: AB_2231974) and whole-brain clearing were 
performed according to previously established protocols (46). For acquisition, cleared samples were imaged in a sagittal orientation (left lateral side up) on a light-sheet microscope (LaVision Biotec) equipped with a sCMOS camera and an LVMI-Fluor $\times 4$ objective lens equipped with a $6 \mathrm{~mm}$ working distance dipping cap. Versions 144 and 210 of Inspector Microscope controller software were used. Samples were scanned in the $640 \mathrm{~nm}$ channel. Images were taken every $6 \mu \mathrm{m}$ and reconstructed with ClearMap software for quantification or with Imaris 9.1 software for visualization. For autofluorescence, the $480 \mathrm{~nm}$ channel was used with a $\times 1.3$ objective lens. Whole-brain tissue was analyzed using ClearMap software (latest version available from https://www.idisco.info/; see also refs. 46,47$)$.

$I H F$. Mice were placed individually in home cages with water and chow ad libitum. For the 2-hour treatment, after 4 days of acclimation, mice were fasted overnight. In the morning (9:00 am), food was added and water was replaced with water supplemented with vehicle or water supplemented with Flx, and mice were euthanized 2 hours later. For the 14-hour treatment, after 4 days of acclimation, mice were treated with water supplemented with vehicle or water supplemented with Flx at 6:45 pm, and chow was replaced with fresh chow (150-170 g). Flx-treated mice were randomized into 3 groups: the first group of mice was euthanized immediately; the second group was euthanized 24 hours after Flx was withdrawn and replaced with vehicle (14 hours of Flx plus 1-day withdrawal); and the third group was euthanized 48 hours after Flx was withdrawn and replaced with vehicle (14 hours of Flx plus 2-day withdrawal). Vehicle-treated mice were also randomized into 3 subgroups and were respectively euthanized at the same time as the Flx-treated or 1- or 2-day withdrawal groups. Since no difference was found between the 3 vehicle-treated subgroups, their data were pooled for analysis. Mice were euthanized between 9:00 am and 11:00 am.

For the withdrawal experiment following long-term treatment, mice were treated with vehicle or Flx for 5 weeks. Then, Flx-treated mice were randomized into 3 groups: 1 group was kept on Flx for 1 more week and euthanized at week 6 from day 0 of treatment; the second group was kept with no treatment for 2 additional weeks and euthanized at week 7 from day 0 of treatment ( 5 weeks of Flx plus a 2-week withdrawal); and the third group was kept with no treatment for 4 weeks and euthanized at week 9 from day 0 of treatment ( 5 weeks of Flx plus a 4 -week withdrawal). Vehicle-treated mice were also randomized into 3 subgroups that were respectively euthanized with the Flx-treated group or with the 2-week or 4-week withdrawal group. Since no difference was found between the 3 control subgroups, their data were pooled for analysis. Mice were euthanized between 9:00 am and 11:00 am.

Euthanized mice were transcardially perfused with PBS followed by $4 \%$ PFA. Brains were then postfixed for 12-24 hours and sectioned to a thickness of $50 \mu \mathrm{m}$ using a floating vibratome. The following primary antibodies were used for immunohistochemical analyses: rabbit c-Fos at 1:1000 (Cell Signaling Technology 2250, RRID:AB_2247211); guinea pig c-Fos at 1:1000 (Synaptic Systems, 226 004, RRID:AB_2619946); rabbit Tph2 at 1:1000 (Novus, NB100-74555, RRID:AB_1049988); rabbit POMC at 1:4000 (Phoenix Pharmaceuticals, H-029-30, RRID:AB_2307442); sheep $\alpha$-MSH at 1:10,000 (MilliporeSigma, AB5087, RRID:AB_91683); rabbit NPY at 1:1000 (Abcam, ab221145); and mouse Htr2c at 1:200 (Santa Cruz
Biotechnology, sc-17797). The following secondary antibodies were used and were Alexa Fluor conjugated: Alexa Fluor 488-donkey antisheep (Invitrogen, Thermo Fisher Scientific, A11015); Alexa Fluor 488-donkey anti-rabbit (Jackson ImmunoResearch, 711-545-152); Alexa Fluor 488-goat anti-guinea pig (Invitrogen, Thermo Fisher Scientific, A11073); and Alexa Fluor 594-donkey anti-rabbit (Jackson ImmunoResearch, 711-585-152). The antibodies were previously validated. All images were captured using a confocal microscope (Zeiss). For the number of cells, the graphs represent the sum of cells counted in 3 slides per mouse and averaged per group. Integrated density values were calculated using Fiji software (48), and the average of 3 slides per mouse is presented.

Cell culturing and in vitro treatments. SH-SY5Y cells (American Type Culture Collection [ATCC]) were cultured in DMEM/F12 10\% FBS and differentiated for 5 days with B-27 Plus Supplement (Gibco, Thermo Fisher Scientific, A3582801); GlutaMAX (Gibco, Thermo Fisher Scientific, 35050061); and ATRA (Abcam ab141962) in Neurobasal Plus Medium (Gibco, Thermo Fisher Scientific, A3582901) prior to SSRI treatment. Flx (Combi-Blocks), paroxetine, fluvoxamine, sertraline, and escitalopram (MilliporeSigma) were dissolved at 10 $\mathrm{mM}$ in $\mathrm{NaCl} 0.9 \%$ and added to the medium at a final concentration of $3 \mu \mathrm{M}$. Cells were treated for 2 hours for Western blotting or for 4 hours for gene expression analysis.

Western blot analysis. Proteins were extracted from cells using PhosphoSafe Extraction Reagent (EMDMillipore). Extract (8-10 $\mu \mathrm{g}$ ) was analyzed by Western blotting using the following antibodies: p-STAT3 (Tyr705) (Cell Signaling Technology, 9131); p-STAT3 (Ser727) (1:1000; Cell Signaling Technology, 9134S); STAT3 (1:1000; Cell Signaling Technology, 9139); GAPDH (1:5000; Cell Signaling Technology, 5174); p-CREB (1:1000; Cell Signaling Technology, 9198); and CREB (1:1000; Cell Signaling Technology, 9104), followed by incubation with HRP-conjugated anti-rabbit IgG (1:2000; Abcam, NA934V) or anti-mouse IgG (1:2000; GE Healthcare, NXA931) antibodies and detection with ECL Western Blot Reagent (Advansta). Imaged blots were quantified using Fiji software (48). For all antibodies, validation is available on the manufacturer's website.

Gene expression. RNA was purified from dissected hypothalami using TRIzol (Invitrogen, Thermo Fisher Scientific). Real-time quantitative PCR (qPCR) was performed using Taq SYBR Green Power PCR Master Mix (Invitrogen, Thermo Fisher Scientific) on a CFX Connect instrument (Bio-Rad). Hprt amplification was used as an internal reference for each sample. Verification of amplicon specificity was tested through a BLAST search, and dissociation curves analysis was performed in every experiment. Primers sequences are listed in Supplemental Table 1 .

Marble-burying test. Twelve glass marbles were evenly spaced in 3 rows, on an approximately $5 \mathrm{~cm}$ layer of sawdust bedding lightly pressed down to make a flat, even surface, in a plastic cage. The day before euthanasia, each mouse was placed in the cage and left for 30 minutes, after which the number of marbles buried with sawdust was counted (49).

Statistics. All values are expressed as the mean \pm SEM. Group sizes were determined by performing a power calculation to lead to an $80 \%$ chance of detecting a significant difference $(P \leq 0.05)$. All values use biological replicates and are indicated by group size $(n)$ in the figure legends and/or graphs plotted with individual values. Statisti- 
cal analyses were performed using 1-way ANOVA or 2-way ANOVA followed by Šidák's or Tukey's test or ANCOVA, to compare means of 3 or more groups, and using an unpaired, 2-tailed Student's $t$ test to compare means of 2 groups, as indicated in the figure legends. All statistical analyses were performed using GraphPad Prism (GraphPad Software), except for the energy expenditure experiments, for which CalR (50) was used.

Study approval. The IACUC of Columbia University approved all procedures.

\section{Author contributions}

MJO and PD conceptualized the study. MJO, MS, and PD designed the study methodology. MJO, MS, AI, KP, and FM performed experiments. MJO, MS, and PD analyzed data. PD acquired funding. Expertise and feedback: JMF and PD provided expertise and feedback. MJO and PD wrote the manuscript.

\section{Acknowledgments}

We thank P. Cohen, G. Karsenty, S. Kousteni, A. Nectow, and L. Zeltser for sharing reagents and equipment, providing advice, and commenting on the manuscript. Images were collected at The Rockefeller University Bio-Imaging Resource Center. This work was funded by the National Institute on Aging (NIA), NIH (AG032959, to PD); the JPB Foundation (to JMF); the Robertson Therapeutic Development Fund (to MS and JMF), the NIH (DK120869, to MS); and a Kavli Neural Systems Institute (NSI) Postdoctoral Fellowship (to MS).

Address correspondence to: Patricia Ducy or Maria Jose Ortuno. Columbia University Medical Center, Black Building, Room BB1412, 650 West 168th Street, New York, New York 10033, USA. Phone: 212.305.9299; Email: pd2193@cumc.columbia.edu (PD); Email: mjo2129@cumc.columbia.edu (MJO).
1. Kane SP. https://clincalc.com/DrugStats/Drugs/ Fluoxetine. ClinCalc DrugStats Database, Version $21.1 ; 2020$.

2. Wong DT, et al. Prozac (fluoxetine, Lilly 110140), the first selective serotonin uptake inhibitor and an antidepressant drug: twenty years since its first publication. Life Sci. 1995;57(5):411-441.

3. Fox M, et al. A pharmacological analysis of mice with a targeted disruption of the serotonin transporter. Psychopharmacology (Berl). 2007;195(2):147-166.

4. Heisler LK, et al. Serotonin reciprocally regulates melanocortin neurons to modulate food intake. Neuron. 2006;51(2):239-249.

5. Xu Y, et al. 5-HT2CRs expressed by pro-opiomelanocortin neurons regulate energy homeostasis. Neuron. 2008;60(4):582-589.

6. Heisler LK, et al. Fluoxetine decreases fat and protein intakes but not carbohydrate intake in male rats. Pharmacol Biochem Behav. 1997;58(3):767-773.

7. Heisler LK, et al. Reduction of fat and protein intakes but not carbohydrate intake following acute and chronic fluoxetine in female rats. harmacol Biochem Behav. 1999;63(3):377-385.

8. Voigt JP, Fink H. Serotonin controlling feeding and satiety. Behav Brain Res. 2015;277:14-31.

9. Burke LK, Heisler LK. 5-hydroxytryptamine medications for the treatment of obesity. JNeuroendocrinol. 2015;27(6):389-398.

10. Jensen-Otsu E, Austin GL. Antidepressant use is associated with increased energy intake and similar levels of physical activity. Nutrients. 2015;7(11):9662-9671.

11. Kivimäki M, et al. Antidepressant medication use, weight gain, and risk of type 2 diabetes: a population-based study. Diabetes Care. 2010;33(12):2611-2616.

12. Lee SH, et al. Is increased antidepressant exposure a contributory factor to the obesity pandemic? Transl Psychiatry. 2016;6(3):e759.

13. Carvalho AF, et al. The safety, tolerability and risks associated with the use of newer generation antidepressant drugs: a critical review of the literature. Psychother Psychosom. 2016;85(5):270-288.

14. Gafoor R, et al. Antidepressant utilisation and incidence of weight gain during 10 years' follow-up: population based cohort study. BMJ. 2018;361:k1951.

15. Andersohn F, et al. Long-term use of antidepressants for depressive disorders and the risk of diabetes mellitus. Am J Psychiatry. 2009;166(5):591-598.

16. Cascade E, et al. Real-world data on SSRI antidepressant side effects. Psychiatry (Edgmont). 2009;6(2):16-18.

17. Lockhart P, Guthrie B. Trends in primary care antidepressant prescribing 1995-2007: a longitudinal population database analysis. $\mathrm{Br} \mathrm{J} \mathrm{Gen}$ Pract. 2011;61(590):e565-e572.

18. Bolo NR, et al. Brain pharmacokinetics and tissue distribution in vivo of fluvoxamine and fluoxetine by fluorine magnetic resonance spectroscopy. Neuropsychopharmacology. 2000;23(4):428-438.

19. Yadav VK, et al. A serotonin-dependent mechanism explains the leptin regulation of bone mass, appetite, and energy expenditure. Cell. 2009;138(5):976-989.

20. Aponte Y, et al. AGRP neurons are sufficient to orchestrate feeding behavior rapidly and without training. Nat Neurosci. 2011;14(3):351-355.

21. Varela L, Horvath TL. Leptin and insulin pathways in POMC and AgRP neurons that modulate energy balance and glucose homeostasis. EMBO Rep. 2012;13(12):1079-1086.

22. Balthasar N, et al. Divergence of melanocortin pathways in the control of food intake and energy expenditure. Cell. 2005;123(3):493-505.

23. Dahlin A, et al. Expression profiling of the solute carrier gene family in the mouse brain. JPharmacol Exp Ther. 2009;329(2):558-570.

24. Bengel D, et al. Altered brain serotonin homeostasis and locomotor insensitivity to 3, 4-methylenedioxymethamphetamine ("Ecstasy") in serotonin transporter-deficient mice. Mol Pharmacol. 1998;53(4):649-655.

25. Ren J, et al. Anatomically defined and functionally distinct dorsal raphe serotonin sub-systems. Cell. 2018;175(2):472-487.

26. Nectow AR, et al. Identification of a brainstem circuit controlling feeding. Cell. 2017;170(3):429-442.

27. Samuels BA, et al. Serotonin $1 \mathrm{~A}$ and serotonin 4 receptors: essential mediators of the neurogenic and behavioral actions of antidepressants. Neuroscientist. 2016;22(1):26-45.

28. Hjorth S, et al. Serotonin autoreceptor function and antidepressant drug action. JPschopharmacol. 2000;14(2):177-185.

29. Hannon J, Hoyer D. Molecular biology of 5-HT receptors. Behav Brain Res. 2008;195(1):198-213.

30. Sprouse JS, Aghajanian GK. (-)-Propranolol blocks the inhibition of serotonergic dorsal raphe cell firing by 5-HT1A selective agonists. Eur J Pharmacol. 1986;128(3):295-298.

31. Gautron L, et al. Neural control of energy balance: translating circuits to therapies. Cell. 2015;161(1):133-145.

32. Ortuno MJ, et al. Serotonin-reuptake inhibitors act centrally to cause bone loss in mice by counteracting a local anti-resorptive effect. Nat Med. 2016;22(10):1170-1179.

33. Oury F, et al. CREB mediates brain serotonin regulation of bone mass through its expression in ventromedial hypothalamic neurons. Genes Dev. 2010;24(20):2330-2342.

34. Berglund ED, et al. Serotonin $2 \mathrm{C}$ receptors in pro-opiomelanocortin neurons regulate energy and glucose homeostasis. J Clin Invest. 2013;123(12):5061-5070.

35. Yeo GS, Heisler LK. Unraveling the brain regulation of appetite: lessons from genetics. Nat Neurosci. 2012;15(10):1343-1349.

36. Quarta C, et al. POMC neuronal heterogeneity in energy balance and beyond: an integrated view. Nat Metab. 2021;3(3):299-308.

37. Bates $S$, et al. STAT3 signalling is required for leptin regulation of energy balance but not reproduction. Nature. 2003;421(6925):856-859.

38. Mosialou I, et al. MC4R-dependent suppression of appetite by bone-derived lipocalin 2. Nature. 2017;543(7645):385-390.

39. Dulawa SC, et al. Effects of chronic fluoxetine in animal models of anxiety and depression. Neuropsychopharmacology. 2004;29(7):1321-1330.

40. O'Leary OF, et al. Chronic fluoxetine treatment increases expression of synaptic proteins in the hippocampus of the ovariectomized rat: role of BDNF signalling. Psychoneuroendocrinology. 2009;34(3):367-381. 
41. Felsing DE, et al. Ligand-directed serotonin 5-HT(2C) receptor desensitization and sensitization. Eur J Pharmacol. 2019;848:131-139.

42. Petropoulou PI, et al. Lipocalin-2 is an anorexigenic signal in primates. Elife. 2020;9:e58949.

43. Greenfield JR, et al. Modulation of blood pressure by central melanocortinergic pathways. $N$ Engl J Med. 2009;360(1):44-52.

44. Haws R, et al. Effect of setmelanotide, a melanocortin- 4 receptor agonist, on obesity in Bardet-Biedl syndrome. Diabetes Obes Metab.
2020;22(11):2133-2140.

45. Clément K, et al. Efficacy and safety of setmelanotide, an MC4R agonist, in individuals with severe obesity due to LEPR or POMC deficiency: single-arm, open-label, multicentre, phase 3 trials. Lancet Diabetes Endocrinol. 2020;8(12):960-970.

46. Renier N, et al. Mapping of brain activity by automated volume analysis of immediate early genes. Cell. 2016;165(7):1789-1802.

47. Renier N, et al. iDISCO: a simple, rapid method to immunolabel large tissue samples for volume imaging. Cell. 2014;159(4):896-910.

48. Schindelin J, et al. Fiji: an open-source platform for biological-image analysis. Nat Methods. 2012;9(7):676-682.

49. Angoa-Perez M, et al. Marble burying and nestlet shredding as tests of repetitive, compulsive-like behaviors in mice. J Vis Exp. 2013;(82):50978.

50. Mina AI, et al. CalR: a web-based analysis tool for indirect calorimetry experiments. Cell Metab. 2018;28(4):656-666. 